\title{
Online Peer Groups as a Persuasive Tool to Combat Digital Addiction
}

\author{
Amen Alrobai, John McAlaney, Keith Phalp, Raian Ali \\ Faculty of Science and Technology \\ Bournemouth University, United Kingdom \\ \{aalrobai, jmcalaney, kphalp, rali\}@bournemouth.ac.uk
}

\begin{abstract}
Digital Addiction (DA) denotes a problematic usage of digital devices characterised by properties such as being compulsive, impulsive, excessive and hasty. DA is associated with negative behaviours such as anxiety and depression. "Digital Detox" programs have started to appear and are mainly based on a relatively expensive and heavyweight in-patient care utilising traditional solutions such as motivational interviews and cognitive behavioural therapies. For moderate addiction, persuasive technology could have potential, as a brief intervention, to assist users to regulate their usage. This paper explores the design of online peer groups as a persuasive technique that puts together people who share a common interest in combating their DA or in helping others to do so. We conducted empirical research to explore design aspects of this mechanism. The results raise a range of questions and challenges to address when developing such a technique for the behaviour change needed against DA.
\end{abstract}

Keywords: Digital Addiction, Digital Health Persuasion, Online Peer Groups

\section{Introduction}

Despite obvious benefits, the emergence of digital technologies and social networking services has also led to negative consequences on modern societies. A recent metaanalysis study covered 80 empirical reports from 1996 to 2012 and concluded that Internet Addiction, which is a form of digital addiction, affects $6 \%$ of people worldwide [1]. In 2013, 3.2\% of British students were considered to be addicted [2]. In South Korea, over 140 Internet Addiction treatment recovery centres opened by 2011 [3]. According to the recent statistics from the China Youth Association for Network Development, the number of Chinese teenage addicts increased to 24 million $(14.1 \%)$ by 2009 [4]. This is over double the number in 2005 , which was around 10 million $(13.2 \%)$. It is worth noting that these studies followed different criteria on assessing addictive usage.

These trends have led to growing interest in research to combat DA using selfregulation systems. These systems are seen as supportive means and emphasize that addicts have an active role in changing their own behaviour. These systems could include for example interactive warning labels containing persuasive techniques like 
timers and avatars to combat DA, as proposed in [5]. In another study [6], a new approach to ICT-facilitated self-regulation was proposed based on social cognitive theory to limit smartphone usage. The approach facilitates creating groups of users to share their usage information. Generally, these systems are based on the assumption that people have the individual ability to adjust and optimise their own behaviour to maximise their gains according to their particular circumstances.

Despite such efforts, the introduction of these software systems to health-related behaviour, including addictive behaviours such as DA, has led to many controversial arguments. Most notable is the lack of strong scientific proof for their potential effectiveness. In a recent study [7], researchers found that many app-based psychological interventions including those hosted by governmental bodies, such as the National Health Service in the UK, fail to demonstrate clinical evidence of a long term change. A longitudinal research study found that delivering interventions within peer group settings could be harmful due to different factors relevant to group structure, which led to reinforcing the negative behaviour [8] such as loafing and compensation [9], and conformity effect [10]. Some negative attributes of persuasive technologies were also reported in [11], such as frustration, anxiety, peer pressure and feeling of guilt for the participants. This suggests a need for further research on the design of such software-based solutions in order to exploit their power whilst attempting to avoid or reduce negative side effects.

In this paper, we introduce online peer groups as a persuasive mechanism based on self-regulation systems, to support an effective and long-term behavioural change to combat DA. This paper conducts an exploratory research on the different aspects that need to be considered when designing online peer groups and reflects on the applicability and potential as well as risks of such a mechanism.

\section{Background and Research Motivation}

A motivational peer group is where people "voluntarily come together to help each other address common problems or shared concerns" [12]. Linking addicts to peer support groups prior to the professional treatment may reduce the duration needed in the initial episodes of treatment and increase recovery rates [13]. Also, extending the participation in peer support groups reduced the need for subsequent treatment episodes [13]. The peer groups approach can also be utilised in the post-treatment to reduce relapse rates [14]. The strength of this approach lies in its distinct persuasive and motivational mechanisms to sustain behavioural change; mainly commitment and consistency, reciprocity, and social proof [15] as well as surveillance which reinforce all these mechanisms. Peer groups technique could utilize the helper-therapy principle [16] which suggests that it can be personally beneficial for addicts to assist others deal with own addictions.

Behavioural change theories such as the theory of planned behaviour [17] and goal-setting theory [18] are used to bridge the gap between attitudes and behaviours. These theories aim at reducing discrepancies between these two conceptual constructs such as, for example, the gap between the intention to change a behaviour and the act of actually doing so [19]. This is achieved by encouraging individuals to create a plan to achieve the targeted behaviour. In self-regulation systems, monitoring is a fundamental design element. It provides a useful basis for effective intervention design by enabling users to track their performance and support them in achieving their goals, whilst also maintaining their regulated behaviours [20]. Self-monitoring is 
a "process of having individuals record data regarding their own behaviour for the purpose of changing its rate" [21]. When doing so, correctly in social settings, such as peer groups, it can further support the positive change.

Goal setting is a key element to guide monitoring processes and make it more meaningful. In DA, it is still a research question as to which type of goals, i.e. proximal and distal, would be more effective to develop self-efficacy and be easier for users to setup. Proximal goals are essential sub-goals to achieve the distal goals. For example, increasing offline social connection is a proximal goal to regulate digital usage and combat DA, the associated distal goal. In DA, we still have no models on the decision rights of goal settings for a particular group and whether it should be set up by the individuals or an authorized moderator or perhaps collectively. Likewise, the decision on setting up the goals is still a research issue, e.g., whether it should be self-set, provider-set, participatory-set, or set up by recommender systems [22].

Self-regulation systems can either monitor behaviour, e.g., the user shared 40 posts this week on their social network, or monitor change in the behaviour, e.g., the user shared fewer posts than last week [23]. It is fundamental to investigate which type of monitoring would motivate users. Some studies, such as [24], concluded that intervention systems for addictive behaviours may fail due to poor application of goal-setting theory, e.g., difficulty in setting standards as well as poor consideration of conflicting goals, such as regulating mobile usage and enjoying the moment, and also distorted goals, such as surfing the Internet to improve mood. These risks pose challenges for group's governance. While there are some successful intervention cases, they are often short term, as such interventions are expensive and hard to maintain [25]. This suggests the need for complementary strategies to support longterm interventions and to reduce relapse rate. This could be achieved through inpatient care, which is expensive and heavy weight for the early stages of DA.

Hence, we suggest investigating a persuasive technique that combines technology with human support to achieve sustainable behaviour change in a flexible and efficient style, hence the suggestion of online peer groups. Peer groups approach can be an appropriate program for users in the transition to addiction stage due to their need for less action-oriented strategies in which immediate change is not expected [26]. It can also benefit those who are also unaware of their level of addiction as it can make them more informed of the consequences occurred to their peers. This paper will explore the potential of online peer groups as a persuasive technique in that regard and focus on different design aspects.

\section{Method and Research Settings}

This paper reports upon work to explore users' perceptions of online peer groups, with respect to their possible use to help digital addicts. We adopted several qualitative methods in two studies to triangulate the findings and to generate more comprehensive understanding.

For the first study, we had a relatively broad remit, to investigate how users would perceive self-monitoring and peer monitoring to combat DA. We first conducted a diary study with 14 participants, 5 male and 9 female, aged between 18 and 50. A convenience-sampling technique was used. A pre-selection questionnaire test was used to ensure that participants had at least one aspect of problematic usage of their smart phones. The pre-selection test was an adapted version of the CAGE questionnaire customized to fit the properties and remit of DA [27]. 
The participants were asked to install one of three commercial digital diet smartphones applications and use it for 14 days, and to record their observations and feelings about the application and their usage style. These diaries were used to guide the follow-up interviews with the same participants. To be selected, the application needed to have a rating of at least three stars out of five, a high number of downloads (no less then 500); at least 5 persuasive techniques, e.g. goal-settings, monitoring reminders, and rewarding, coercion and surveillance. This was to allow users to engage with a wide range of persuasive features in order to assess their influence and suitability for DA. After 14 days of usage and writing reflections and feedback, we conducted semi-structured interviews with the same participants to elaborate on their diaries. One of the interviews questions was about the social features of the applications, which represent various aspects of online peer groups.

The second study focused on understanding different perspectives on online peer groups and their interactive design. We conducted a two sessions focus group study. The first one included 6 participants, 3 males and 3 females. The participants had preexisting social relationships with each other so they aligned with the concept of a peer group, which requires some degree of shared interest and trust. Ages ranged between 20 and 26. The CAGE-like questionnaire was again used as a pre-selection test. Participants were given an engaging task in which they had to comment on and construct different online peer group designs and interaction styles including the use of persuasive techniques to regulate addiction.

We used all obtained data in the first session to come up with a new peer group design and made it the subject of discussion in the next session in which the same participants would engage as potential users of such online peer group aiming to regulate their addiction. However, one of the participants, who could not participate, was replaced with another one who met the selection criteria and had good experience in 3D animation, which was advantageous to give some ideas on a creative design.

Finally, we performed a survey study to get further confirmation and insights through comments from a wider sample on our findings. A total of 73 completed responses were returned from the sample of 42 male and 31 female, aged between 18 and 65 , recruited through an open call via several academic mailing lists.

In order to scope our analysis and as an initial template, we used the Cialdini's six principles of influence [15] to investigate the potential influential aspects in social settings from users' perspective as well as Fogg's behavioural model [28] to focus on the technology-facilitated features that can maximise the persuasion of online peer groups.

\section{Results}

Different aspects and areas of concerns about peer groups have been explored such as the study in [8]. This includes the personal characteristics that can heavily influence the effectiveness of ex-addicts and non-professional participation in counselling activities [29]. In online peer groups, such concerns need to be revisited by exploring what digital addicts prefer in terms designing online peer groups. Fig. 1 presents the main aspects of online peer groups for DA. This conceptual map reflects the areas of concerns that are considered important from users' perspective. Governance, Structuring and Moderation will be discussed in separate subsections while Risks will be discussed as a cross-cutting aspect through all subsections. 


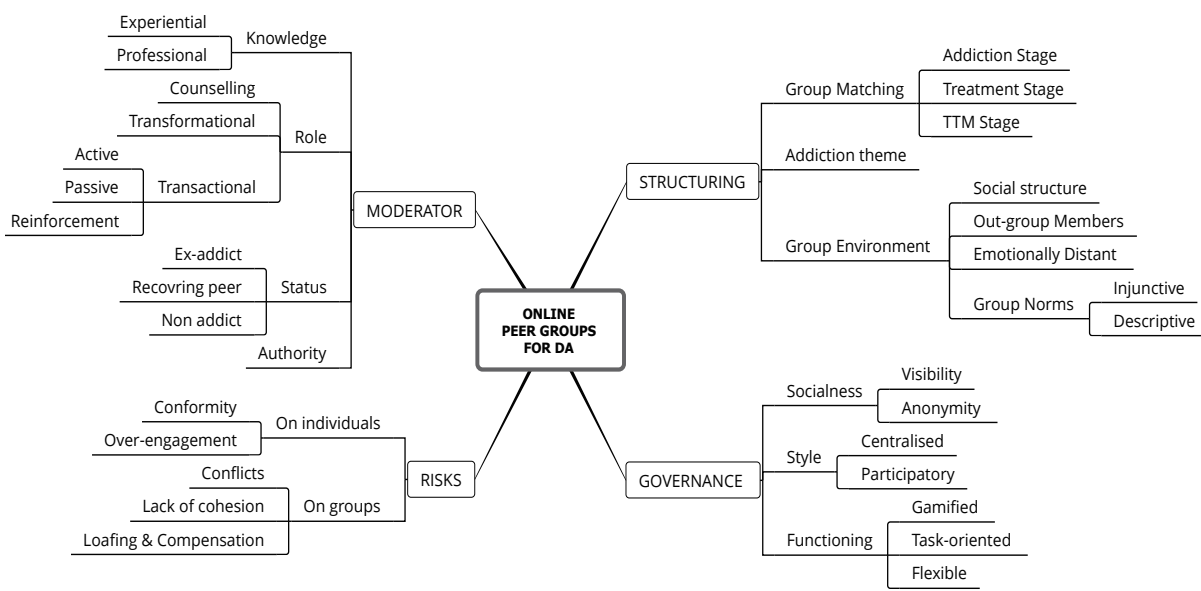

Fig. 1. Online peer groups for digital addiction

In the next subsections, we present general findings including the usefulness of peer groups to regulate DA, users' motivations to join them and how group structuring is seen and preferred by the users. We will also discuss various concerns and design issues related to the role of groups' moderators and the application of behaviour change theories and persuasive techniques within social settings including social norms.

\subsection{Attitudes to the Overall Concept of On-line Peer Groups}

In our study, we assessed the perception of digital addicts of the usefulness of peer groups to regulate their usage. The overall impression was positive, and $71 \%$ felt that a peer group would be useful (26\% certainly, $45 \%$ somehow). Participants liked the idea of technology-limiting technology and found "online system to cure online addiction is an interesting concept". However, it had not escaped the authors, nor had it escaped some of the participants, that there is a paradox in supporting online addiction by inviting people to partake of online support, as part of another community. Perhaps one of the comments that highlight this best was one person who likened the approach "inviting alcoholics down the pub to chat about their alcoholism". Of course, there are key differences here. Firstly, the medium, the online, is for many, something that they cannot choose to avoid. As such, being connected is a fundamental part of their life, or indeed, for many, their professional life. Secondly, digital addiction is a software-mediated behaviour. Hence, with the aid of software means, it is possible to actively monitor and intervene when necessary.

To identify the primary appealing characteristics of online peer groups, participants were asked about their motivation to join peer groups. Interestingly, most of the comments were clearly stating that providing moral support to others would be the main motivation. This motivation can be triggered by reciprocity norm [15] which suggests that the equality of power and exchanging help are the essential ingredients to build effective groups. This is well understood in the "helper therapy" principle [16]. That is, participants find it more motivating to be also useful for others, not only addicts who are seeking for help. This is perhaps the key element of peer groups. 
Some other techniques used in persuasive systems seem not to be an important aspect for online peer groups used to assist the behavioural change for combating DA. For example, the Cialdini's Liking principle suggests that people are easily influenced by those who they admire, such as celebrities. That is to say popular persuasive techniques may not be seen efficient and even accepted when applied for behavioural change in addictive behaviours, such as DA, and they need to be revisited for that context of use.

\subsection{The Moderation Role in Online Peer Groups}

Participants highlighted the important role of having the human element such in software systems advocating techniques like peer groups to build a sense of trust and commitment to support long-term change. For example, one participant commented that in young age groups, having parental involvement would benefit more as they have a sense of authority to regulate the usage. For all of the individuals within the group, the role of the moderator was clearly understood as of paramount importance.

For example, there was a legitimate concern that there is a high risk factor of peers developing deviant behaviours due to normative influence [30]. This is one of the main reasons to introduce the moderation as an essential process in online peer groups. Moderator can also play other typical governance roles such as those related to memberships and rewards allocation as well as addressing the influence of the nonmatched members such as grouping members who belong to different levels of change.

\section{Characteristics of Moderators}

Participants were asked to consider a range of potential characteristics for the crucial role of moderator. Of these, only one question gained greater than $50 \%$ agreement, $58 \%$ believing the moderator must be "accredited / professional / should be professional for [advanced digital] addiction". The comment above appear to endorse the need for a 'professional' moderator, though an exception was highlighted when groups or moderators have "successful support history" regardless of their professional knowledge. Thus, the experiential knowledge was perceived as an appealing attribute of the moderation role. In addition, two further clear themes are clear from the comments.

The first observation is that the participants made a distinction between 'light' and 'advanced stage' digital addiction. That is, they suggest that for early stage addiction, friends or less qualified people might be helpful in a peer group, and this further suggests that such a peer group approach as an early, low cost, intervention is something that they consider to be useful even among genuine peers. Two comments exemplify this commonly stated view: 1) "for groups with advanced addiction, moderator should be a therapist or a digital addict or an ex digital addict but with therapist expertise so they know what to say and how to say it", and 2) "For light addiction anyone really, does not matter, I would say the same for early addiction that means to prevent it first or to recover from it. I see difference".

The second observation is that the attitude or approach of the moderator is seen as paramount, friendliness and liberal styles being mentioned, one argued that "friendliness in the group is a main requirement". However, again these comments being qualified by those cases where 'professional treatment', requires a therapist.

Finally, the question of whether moderators should themselves be ex-addicts drew far more mixed response. While some, $20 \%$ of the survey, considered that ex-addicts 
would have more empathy, a greater number suggested that the moderator should not be an ex-addict, "addicts might dictate their opinion and be biased to their own experience".

\section{The Role of Moderators}

Participants wanted to have collaborative moderators, who have the ability to guide the behavioural change by providing inspirational motivation. For example, a participant commented that a moderator should be "someone who is respectable and can take charge; but also sympathises". The most positive responses for activities of the moderator, were: create and suggest rules (of engagement) 58\%, support motivation (55\%), provide advice to members $(54 \%)$, and create real life events $(54 \%)$ - but of course this final suggestion contrasts with anonymity, and reward members for complaint behaviour / usage (though note this is taken on trust) $50 \%$.

Hence, the moderator's role was seen, as in other forms of addiction, as primarily about setting out and controlling how people interacted, suggesting rules, motivating members, and giving advice. A perhaps surprising finding is that many wanted moderators to create real life events, contrasting with other questions for anonymity. Finally, while the moderator giving some kind of rewards was favoured by $50 \%$ of the survey respondents, penalties was, a much lower score, with only $30 \%$ believing that the moderator should give penalties. An interesting comment from the survey, which again tallied with our other studies was: "No penalty but probably confrontation with their status", since one of the perceived benefits is concrete evidence and heightened awareness of actual usage. These characteristics of the role of moderation align well with the transformational leadership paradigm [31].

The use of "rational or economic means" to strengthen the probability of members' compliance with group's goals, suggests a moderation role that follows transactional leadership paradigm [31]. In this type of moderation, two approaches can be taken. The first is about active moderation and requires monitoring groups' interaction to ensure continued enhancement of the performance through applying corrective actions. Second is a passive moderation in which a moderator intervenes and applies operant conditioning when group's goals and standards are violated [31]. Other users preferred to have the moderator as a councillor, so members request their interventions when needed. As such, no monitoring and direct intervening are required.

Using persuasive techniques for behavioural change might lead some design issues related to the moderator role in peer groups. Participants argued that in some groups a moderator should be enabled to guide the change through positive reinforcements and light penalties as persuasive techniques. For example, one participant commented: "people may leave a peer group if too much penalty is enforced". Another one highlighted that if penalties must be implemented should take more influential approach such as "confrontation members with their status".

The careful implementation of persuasion techniques and moderation role will have profound a impact on groups' self-esteem. One of main components in Fogg's model for persuasive design [28] is the ability to perform the targeted behaviour or to reduce negative behaviour. When reducing the negative behaviour is very challenging goal such as in severe addiction, we would expect the design of online peer groups to increase the motivational influence and to apply the right triggers. This is to increase the probability of behavioural change to occur. For example, providing means to express the confidence in member's ability to change or applying the right social norm would increase the perceived self-efficiency which will act as 
a powerful motivational tool [32]. Peer group design should provide moderators with means to enact such policies and enable an effective persuasion to change behaviour.

\subsection{Structuring of Online Peer Groups}

Professional involvement in severe addiction cases suggests that the stage of addiction, i.e. early, intermediate and advanced, has an influence on how to customise online peer groups. Thus, we would expect the design on online peer groups to sense users' addiction status and adapt different facets of this mechanism accordingly to provide more persuasive effects.

Ultimately, each stage of addiction represents different level of self-control and distinct attitudes and behaviours. Regardless of the extent to which the object of addiction dominates decision-making processes, individuals with less severe addictive behaviour can be guided through the stage of change [26]. The stage of change, correspond to the stages of the Transtheoretical model (TTM) to behavioural change [26].

A critical assumption is that persuasive software-mediated interactions are more suitable for those who are open to the change, honest and do not have denial of reality. On the other hand, users who exhibit severe addiction symptoms require different course of action and more comprehensive treatment regardless of the stage of change they are at [26]. However, peer groups can still play a role in different phases of that comprehensive treatment, e.g. pre-treatment phase to support problem recognition "non-addicts have no idea but they may give a perspective and may learn how it feels" and post-treatment phase to support relapse avoidance as highlighted in [13]. Structuring peer groups should also consider the theme of addiction. For example addiction to online pornography would require certain degree of anonymity. This aspect will be discussed in the next section.

The social structure within online peer groups seems a very important aspect to be considered in the design. For example, a participant commented "friends are not always the good thing here but unknown people with no direct contact or a friend of friend might be better and more relaxed" and he continued, "family members would be distracting in the group as I may need to behave differently". Only, 9\% of the responses were in favour of having family members. However, another one commented: "family members are fine to have in the online peer groups but not as moderators". This suggests that the design needs to consider the impact of including family and friends versus unknown individuals in the group. This could be linked to the severity and domain of DA. Thus, the design of online peer groups must also consider the domain of addictions, such as gambling and pornography, which would require higher level of anonymity.

The social norms approach has become a major focus of research in recent years and is widely adopted in different developmental sectors such as educational settings in the United States [33]. The approach has been successfully used for behavioural change in the domain of addiction as well as a number of health and socially relevant behaviours [33].

As has been demonstrated extensively throughout social psychological research, individuals are strongly motivated to alter their own thoughts and behaviours to match the norms of the group [34]. This can include descriptive norms, which refers to how often or extensively we perceive our peers to engage in a behaviour or injunctive norms, which refers to the attitudes we believe our peers to hold. In a case of reciprocal causality individuals will also seek out social groups whose behaviours and 
attitudes they perceive to reflect their own [35]. Explicit attempts to manipulate groups, particularly by out-group members, can lead what known as a reactance response in which individuals engage even more strongly in the original behaviour [36]. However people also tend to underestimate how easily influenced they are by the groups they belong to [37]. As such by challenging the perceived norms within a group or encouraging the group to aspire towards a healthier norm behaviour change may be achieved.

Research into the use of peer networks to bring about behaviour change would suggest that they can indeed create new and more positive social norms [38]. There is overall though a lack of research on how social norms may operate within online peer groups.

The degree to which social norms may operate differently in online groups could be expected to reflect the complexity of the social relationships between the members of the peer groups. In the case of peer groups where members feel emotionally distant from one another they are less likely to conform to the norm [39]. However conformity to the social norm is more likely to occur in groups where there is a shared sense of a common goal and a belief that each member plays an important role in the achievement of this goal [10]. If peer groups are therefore to be used to address DA it is important that this is done in a way that engages the group, creates an agreed norm to aspire too and involve all the members of this group into the process. A participant commented, "if a group of people I knew were all trying to cut down their phone usage then I think it would [motivate me to cut down my usage".

\subsection{Governance and Social Aspects in Online Peer Groups}

Enabling computer-mediated interactions among peers raises several social-related concerns. Participants highlighted the level of anonymity as key motivation to join peer groups. Several participants commented that such platforms should be a "safe space" in which users can maintain certain level of anonymity not necessarily complete, as self-disclosure is a key aspect in such social software platforms. For example, anonymity might need to be maintained at the level of members' interactions only, i.e. a member cannot be identified by other members but still identifiable by the system to monitor his progress over time. As such, online peer groups should accommodate various degrees of anonymity [40]. We expect the design of online peer groups to consider the addiction theme as an important aspect in deciding the suitable levels of privacy. More work is still needed to look into how addicts perceive anonymity and the influential privacy aspects that plays a role in persuading them to join and sustain their participation.

The experiential knowledge attribute mentioned in section (4.2) suggests visibility concept in groups functioning as an important persuasive feature to enhance the intragroup's trust. Participants used the visibility term to denote the notion of having accessible service history and overall performance of groups and moderators. In this sense, anonymity and visibility are not conflicting requirements as the latter revolves around participation visibility rather than participant's visibility.

Other participants showed interest in sharing the role of moderator to maximise group's outcomes. The observation from the lack of having a particular trend in electing moderators is perhaps due to people in peer groups wanting a participatory style and to hear from peers rather than authority figures, such as parents, which take more centralised style. 
As with the whole concept of using online to regulate DA drew some mixed views, and many noted the apparent paradox of having gamification and online approaches due to the risk from over-engagement. However, on the whole participants were positive about bringing some 'fun' to the peer group, and transfer of activities is often something useful within traditional addictions. On the whole, competition inside the group was seen as potentially problematic whereas they wanted to support rather than to compete other members. However, from all stages of the research there was a mention of the possibility of the group having an overall usage, from all members, and that they might wish to see this as a collective goal, or even compete with other groups. Clearly, this is an often used gamification tactic, call centre teams compete with each other, and often weight loss has been tackled with such an approach. Having the element of competition suggests that the group functioning can take taskoriented approach to either meet individuals' or collective goals. Other users wanted more flexible and supportive medium that is free from competition to "support rather than beat each other".

In terms of specific tactics, 53\% wanted points for compliance with 'healthy' activity, whereas only $40 \%$ were happy with the idea of something like a leader board. Of course one person noted the monitoring issue, stating "points are good but how will you monitor off-line behaviour?"

Finally, and perhaps to be expected for peer support, the most desirable feature, $(64 \%)$, was online chat, further reinforcing the impact of people in support.

\section{Conclusions and Future Work}

In this paper we explored different aspects of online peer groups, as a motivational mechanism, from users perspectives. We demonstrated its prominent persuasive considerations. Although peer groups technique aims at supporting individuals in all addiction levels, we argue the need for careful re-evaluation of the online version of it from the perspectives of behavioural change theories. We also argue that tailoring such social software platform to support those with advanced stage addiction would be a very challenging task. Users who exhibit severe addiction are more vulnerable to relatively unconscious distorted, conflicting, changing requirements and could be accompanied with denial of reality. Our understanding of how social norms operate within peer groups is based primarily on offline interactions. Online environments may have unique characteristics that need to be better understood if change is to be achieved.

The current state of classical methods in software development, e.g. requirements engineering, is not efficient enough to deal with users in that stage. Thus, to design persuasive systems for DA, future studies are required to re-visit software engineering methods to customise existing elicitation models to the domain of behavioural addiction. Ultimately, adapting online peer groups to different users' needs and expectations should eventually produce more persuasive effects. Using simple metrics such as time and frequency to measure the level of addiction would perhaps provide misleading assertions. As such, we argue that measurement models should consider the psychological research on the addiction severity based on clinical criteria, such as salience, conflict and relapse [41]. Thus, users feedback should feed into future measurement models to provide meaningful and suitable configurations for online peer groups. The design of such user feedback acquisition and its peculiarities in this domain, e.g. to detect and react against denial of reality, is a challenge to address. 


\section{Acknowledgment}

The research was supported by Bournemouth University through the PGR Development fund. We would like also to thank Asad Khan and Yasmeen Abdalla for their valuable contributions in conducting the focus group and diary studies in the early stages of this research.

\section{References}

1. Cheng, C., Li, A.Y.-L.: Internet Addiction Prevalence and Quality of (Real) Life: A MetaAnalysis of 31 Nations Across Seven World Regions. Cyberpsychology, Behavior, and Social Networking. 17, 755-760 (2014).

2. Kuss, D.J., Griffiths, M.D., Binder, J.F.: Internet addiction in students: Prevalence and risk factors. Computers in Human Behavior. 29, 959-966 (2013).

3. Young, K.S., de Abreu, C.N.: Internet Addiction: A Handbook and Guide to Evaluation and Treatment. (2011).

4. China Youth Association for Network Development: Young Internet addicts on the rise, http://www.chinadaily.com.cn/china/2010-02/03/content_9417660.htm.

5. Ali, R., Jiang, N., Phalp, K., Muir, S., McAlaney, J.: The Emerging Requirement for Digital Addiction Labels. REFSQ. 9013, 198-213 (2015).

6. Ko, M., Yang, S., Lee, J., Heizmann, C., Jeong, J., Lee, U., Shin, D., Yatani, K., Song, J., Chung, K.-M.: NUGU: A Group-based Intervention App for Improving Self-Regulation of Limiting Smartphone Use. Presented at the February (2015).

7. Leigh, S., Flatt, S.: App-based psychological interventions: friend or foe? Evidence Based Mental Health. (2015).

8. Dishion, T.J., McCord, J., Poulin, F.: When interventions harm: Peer groups and problem behavior. American Psychologist. 54, 755-764 (1999).

9. Karau, S.J., Williams, K.D.: Social loafing: A meta-analytic review and theoretical integration. Journal of Personality and Social Psychology. 65, 681-706 (1993).

10. Allen, V.: Situational factors in conformity. Advances in experimental social psychology (1965).

11. Hamari, J., Koivisto, J., Pakkanen, T.: Do Persuasive Technologies Persuade? - A Review of Empirical Studies. PERSUASIVE. 8462, 118-136 (2014).

12. Davidson, L., Chinman, M., Kloos, B., Weingarten, R., Stayner, D., Tebes, J.K.: Peer Support Among Individuals With Severe Mental Illness: A Review of the Evidence. Clinical Psychology: Science and Practice. 6, 165-187 (2006).

13. Moos, R.H., Moos, B.S.: Help-seeking careers. Journal of Substance Abuse Treatment. 26, $167-173$ (2004).

14. Moos, R.H., Moos, B.S.: Paths of Entry Into Alcoholics Anonymous: Consequences for Participation and Remission. Alcoholism: Clinical \& Experimental Research. 29, 18581868 (2005).

15. Cialdini, R.B.: Influence. HarperCollins (2009).

16. Riessman, F.: The "Helper" Therapy Principle. Social Work. 10, 27-32 (1965).

17. Ajzen, I.: The theory of planned behavior. Organizational behavior and human decision processes. (1991).

18. Locke, E.A., Latham, G.P.: A theory of goal setting \& task performance. (1990).

19. Webb, T.L., Sniehotta, F.F., Michie, S.: Using theories of behaviour change to inform interventions for addictive behaviours. Addiction. 105, 1879-1892 (2010).

20. Torning, K., Oinas-Kukkonen, H.: Persuasive system design: state of the art and future directions. PERSUASIVE. 30 (2009).

21. Coleman, M.C., Webber, J.: Emotional and Behavioral Disorders: Theory and Practice. 
Allyn \& Bacon (2002)

22. Strecher, V.J., Seijts, G.H., Kok, G.J., Latham, G.P., Glasgow, R., DeVellis, B., Meertens, R.M., Bulger, D.W.: Goal Setting as a Strategy for Health Behavior Change. Health Educ Behav. 22, 190-200 (1995).

23. Maitland, J., Chalmers, M.: Self-monitoring, self-awareness, and self-determination in cardiac rehabilitation. CHI. 1213-1222 (2010).

24. Vohs, K.D., Baumeister, R.F.: Handbook of Self-Regulation, Second Edition. Guilford Press (2013)

25. Green-Demers, I., Pelletier, L.G., Ménard, S.: The impact of behavioural difficulty on the saliency of the association between self-determined motivation and environmental behaviours. Canadian Journal of Behavioural Science/Revue canadienne des sciences du comportement. 29, 157-166 (1997).

26. Prochaska, D.J.O.: Transtheoretical Model of Behavior Change. In: Gellman, M.D. and Turner, J.R. (eds.) Encyclopedia of Behavioral Medicine. pp. 1997-2000. Springer New York, New York, NY (2013).

27. Ewing, J.A.: Detecting alcoholism: the CAGE questionnaire. Jama. (1984).

28. Fogg, B.J.: A behavior model for persuasive design. PERSUASIVE. 40 (2009).

29. Snowden, L., Cotler, S.: The effectiveness of paraprofessional ex-addict counselors in a methadone treatment program. Psychotherapy: Theory, Research \& Practice. 11, 331-338 (1974).

30. Allen, J.P., Chango, J., Szwedo, D., Schad, M., Marston, E.: Predictors of Susceptibility to Peer Influence Regarding Substance Use in Adolescence. Child Development. 83, 337-350 (2011).

31. Bono, J.E., Judge, T.A.: Personality and Transformational and Transactional Leadership: A Meta-Analysis. Journal of Applied Psychology. 89, 901-910 (2004).

32. Shamir, B., House, R.J., Arthur, M.B.: The Motivational Effects of Charismatic Leadership: A Self-Concept Based Theory. Organization Science. 4, 577-594 (1993).

33. McAlaney, J., Bewick, B., Hughes, C.: The international development of the "Social Norms" approach to drug education and prevention. Drugs: education. 18, 81-89 (2011).

34. Kelman, H.C.: Interests, Relationships, Identities: Three Central Issues for Individuals and Groups in Negotiating Their Social Environment. Annu. Rev. Psychol. 57, 1-26 (2006).

35. Ennett, S.T., Bauman, K.E.: The contribution of influence and selection to adolescent peer group homogeneity: The case of adolescent cigarette smoking. Journal of Personality and Social Psychology. 67, 653-663 (1994).

36. Fuegen, K., Brehm, J.W.: The intensity of affect and resistance to social influence. Resistance and persuasion. (2004).

37. Darley, J.M.: Social organization for the production of evil. Psychological Inquiry. 3, 199218 (1992).

38. Wright, K.B., Bell, S.B., Wright, K.B., Bell, S.B.: Health-related Support Groups on the Internet: Linking Empirical Findings to Social Support and Computer-mediated Communication Theory. J Health Psychol. 8, 39-54 (2003).

39. Greene, J.D., Sommerville, R.B., Nystrom, L.E., Darley, J.M.: An fMRI investigation of emotional engagement in moral judgment. Science. (2001).

40. Kobsa, A., Schreck, J.: Privacy through pseudonymity in user-adaptive systems. TOIT. 3, 149-183 (2003).

41. Griffiths, M.: A "components" model of addiction within a biopsychosocial framework. J Subst Use. 10, 191-197 (2005). 